\title{
El impacto de las enfermedades fúngicas en el cultivo de soja (Glycine max) en Paraguay ¿es posible estimar las pérdidas?
}

\author{
The impact of fungal diseases on soybean (Glycine max) crops in \\ Paraguay. Is it possible to estimate the losses?
}

\begin{abstract}
Andrea Alejandra Arrua ${ }^{* 1,2}{ }^{\circ}$, Horacio D. Lopez-Nicora ${ }^{3}$, Danilo Fernández Ríos ${ }^{2}{ }^{\circ}$, Cristhian Javier

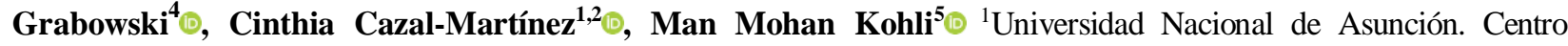
Multidisciplinario de Investigaciones Tecnológicas. San Lorenzo, Paraguay ${ }^{2}$ Universidad Nacional de Asunción. Facultad de Ciencias Exactas y Naturales. San Lorenzo, Paraguay. ${ }^{3}$ Universidad San Carlos. Clínica Vegetal de la Universidad San Carlos. Asunción, Paraguay. ${ }^{4}$ Universidad Nacional de Asunción. Facultad de Ciencias Agrarias. San Lorenzo, Paraguay. ${ }^{5}$ Cámara Paraguaya de Exportadores y Comercializadores de Cereales y Oleaginosas CAPECO. Asunción, Paraguay. *Autor de correspondencia: andrea.arrua@cemit.una.py.
\end{abstract}

Recibido: 30 abril 2021

Aceptado: 14 junio 2021

Recibido en versión modificada: 9 junio 2021

Arrúa, A. A., Lopez-Nicora, H. D., Fernández Ríos, D., Grabowski, C. J., Cazal-Martínez, C. C. \& Kohli, M. M. (2021). El impacto de las enfermedades fúngicas en el cultivo de Soja (Glycine max) en Paraguay ¿Es posible estimar las pérdidas? Investigaciones y Estudios - UNA, 12(1), 34-46. https://doi.org//10.47133/IEUNA2114

Resumen. La economía del Paraguay es altamente dependiente de la producción agrícola, en especial de la soja (Glycine max), por tanto, todo aquello que represente un riesgo para este cultivo es un asunto prioritario para el país. Las fuentes principales de riesgo en la agricultura derivan de los impactos en la producción por el clima, plagas y otros factores en precios y en el contexto institucional y político. La estimación de las pérdidas de los cultivos es fundamental, ya que cuantificar los daños y compararlos con los umbrales económicos permite una adecuada toma de decisiones, determinando si un tratamiento es o no necesario y cuándo; qué se hará el próximo año agrícola, la selección de las variedades, el tipo de manejo que se hará de las enfermedades, entre otros. Considerando que los agentes fúngicos pueden resultar una amenaza para la producción, el objeto de esta revisión fue determinar si existen de manera pública datos para estimar pérdidas a causa de enfermedades fúngicas en soja (G. max). Se concluye que con la información disponible actualmente no es posible determinar estimar las pérdidas y se visualiza la necesidad de generar datos válidos y fidedignos, además de realizar un trabajo coordinado entre productores, industria, gobierno y la academia.

Palabras clave. cultivos extensivos, epifitias, enfermedades fúngicas, Paraguay, pérdidas.

Abstract. Paraguay's economy is highly dependent on agricultural production, especially soybeans $(G$. max), therefore, everything that represents a risk for this crop is a priority issue for the country. The main sources of risk in agriculture derive from the impacts on production due to climate, pests and other factors in prices and in the institutional and political context. 
Estimating crop losses is essential, since quantifying the damage and comparing it with economic thresholds allows adequate decision-making, determining whether a treatment is necessary and when, wthat will be done next agricultural year, the selection of varieties, the type of disease management to be applied, among others. Considering that fungal agents can be a threat to production, the purpose of this review was to determine if public data exists to estimate losses due to fungal diseases in soybeans $(G$. max). We concluded that with the information currently available it is not possible to estimate the losses and that it is necessary it is necessary to generate valid and reliable data, in addition to carrying out coordinated work between producers, industry, government, and academia.

Keywords. extensive crops, epidemics, diseases, Paraguay, losses.

\section{INTRODUCCIÓN}

La agricultura representa el principal factor de crecimiento económico del Paraguay, sin embargo, se ve expuesta a una serie de riesgos que pueden afectar a la producción y comercialización de los productos y que generan volatilidad afectando negativamente a la economía (Abc Color, 2019). El informe del Banco Mundial señala que la agricultura es un sector clave de la economía paraguaya (30 \% del PIB y $40 \%$ de las exportaciones), por lo cual los riesgos agropecuarios tienen repercusiones sobre el crecimiento de la economía, las finanzas públicas, el desempeño de las cadenas de valor y la pobreza rural (Banco Mundial, 2014).

La intensidad y frecuencia de las amenazas determinan su importancia para el sector. La Organización para el Desarrollo Económico y la
Cooperación (OCDE en inglés) señala que principales fuentes de riesgo en la agricultura derivan de los impactos en la producción (por el clima, plagas $\mathrm{u}$ otros factores), en precios (mercados), y en el contexto institucional y político, sin distinción entre países (FAO \& IICA, 2017). El Banco Mundial, describe detalladamente tres grupos principales de riesgos asociados a la agricultura en nuestro país, Riesgos de producción: La producción de granos en Paraguay está expuesta principalmente a riesgos de tipo agroclimático y en menor medida a la incidencia de plagas; riesgos de mercado. La volatilidad de los precios de exportación y de los precios domésticos; riesgos de contexto relacionados con las exportaciones, que en gran medida repercuten en mayores costos de comercialización y que incluyen por ejemplo la inseguridad en la oferta de barcazas para el transporte fluvial hasta los puertos de ultramar, variaciones en los costos del transporte en barcazas en distintos períodos del año, variación de los costos de los fletes internos e inseguridad logística por el mal estado de las rutas y la falta de pavimentación de algunas de ellas en zonas de gran importancia productiva entre otros (Banco Mundial, 2014).

Dentro de los rubros agrícolas, la soja ( $G$. max) es el más relevante en el Paraguay. En 2020 se alcanzó un récord de producción con 10.680.000 Tn y un rendimiento medio de $2.960 \mathrm{~kg} / \mathrm{Ha}$ (Abc Color, 2020). En 2018, la producción de soja alcanzó 10.262.575 toneladas (Tn), sin embargo en 2019 disminuyó a 8.512.008 Tn, una reducción de 1.750.567 Tn (CAPECO, 2021a). Estas pérdidas se relacionaron a diferentes factores climáticos desfavorables: siembras tempraneras $\begin{array}{lll}\text { (aproximadamente } \quad 1.000 .000 & \text { Ha) debido }\end{array}$ principalmente a temperaturas frescas, exceso de 
lluvia y pocas horas luz, que produjeron plantas con poco desarrollo y disminución de los rendimientos; siembras de ciclo medio (aproximadamente 1.300.000 Ha) sufrieron un déficit de lluvias al momento de llenado de granos, aun así se presentaron los mejores rendimientos; y siembras tardías (aproximadamente 1.244.000 Ha), presentó pérdidas por las temperaturas elevadas, déficit hídrico, aborto de flores y vainas, y el rendimiento fue intermedio (CAPECO, 2019b).

En los últimos 5 años, el área de siembra de soja ( $G$. max) ha aumentado en un $7 \%$ y la producción se ha mantenido cercana a los 10 millones de toneladas, con una media de 9.715.693 \pm 730.479 con un rendimiento de $2825 \pm 224 \mathrm{Kg} / \mathrm{Ha}$. Durante la campaña 2018-2019, registró un quiebre de la producción de 23,5\% asociado principalmente a factores climáticos (CAPECO, 2019a).

En su informe titulado "Paraguay, notas de política 2018”, se mencionan 3 riesgos principales relacionados a la agricultura (Banco Mundial, 2018): 1. Los precios de los mercados, señalando que Paraguay cuenta con pocos productos pasibles de exportación y que además se exportan a pocos mercados; sumado a esto, existe una alta dependencia de los precios internacionales de estos productos; 2. Los brotes de enfermedades, que ocasionan pérdidas al productor y a la economía nacional y que pueden llevar al cierre de los mercados; 3. Los eventos climáticos extremos, ya que la agricultura es altamente dependiente del clima y que a su vez pueden afectar la salida de los productos por la hidro vía.

En cuanto a enfermedades asociadas a soja $(G$. max), en Paraguay, se ha mencionado la presencia 33 especies (INBIO, 2016; Ramirez \& Orrego, 2008; Sato et al., 1993; Scholtz, 2020; Xavier et al., 2013;
Yorinori et al., 2005). Entre los hongos fitopatógenos, se destacan Phakopsora pachyrhizi y Macrophomina phaseolina, capaces de causar grandes pérdidas en el cultivo (INBIO, 2016; Scholtz, 2020).

La estimación de las pérdidas de rendimiento causadas por enfermedades se puede realizar por métodos directos e indirectos. Los métodos directos incluyen: a) Cuantificar el patógeno o enfermedad, b) Generación de epidemias de diferente duración e intensidad, c) Experimentos para proporcionar datos para caracterizar la relación enfermedad-pérdida, d) Modelado y e) Estudios de campo. Los métodos indirectos por otra parte, incluyen: a) Testimonio de expertos b) Consultas y encuestas c) Revisiones de literatura d) Experimentos de campo que incluyan ensayos con fungicidas, ensayos de variedades e) Uso de sensores remotos (Teng, Mew, Bonman, \& Leung, 1989).

Por otra parte, las pérdidas económicas debidas a enfermedades pueden subestimarse fácilmente.

Los costos asociados con el diagnóstico y manejo de enfermedades son gastos de producción que a menudo no se incluyen en las estimaciones económicas de pérdidas debidas a enfermedades.

Los costos relacionados con la enfermedad incluyen el tiempo de monitoreo de los campos (horas hombre), honorarios por servicios de consultoría o diagnóstico, y costos asociados con diagnósticos erróneos (Allen et al., 2017).

Por otra parte, la gestión de varias enfermedades de la soja incluye resistencia de cultivares, rotación de cultivos, labranza, fungicidas y manejo de insectos / vectores y plantas hospedantes alternativas entre otros (Allen et al., 2017).

De ahí surgen las preguntas: ¿qué tipo de trabajos, informaciones, datos se han publicado sobre enfermedades fúngicas en soja en Paraguay? ¿es 
posible determinar la existencia y fiabilidad de los datos necesarios para la estimación de pérdidas de rendimiento de soja a causa de enfermedades fúngicas a partir de los informes disponibles y de acceso público? Este trabajo se propuso determinar la existencia de datos fiables y de acceso público que permitan la estimación de pérdidas debido a enfermedades fúngicas en el cultivo de soja.

\section{MATERIALES Y MÉTODOS}

Se realizó una búsqueda bibliográfica en bases de datos especializadas tanto científicas como técnicas, incluyendo, el Centro de Información Científica del CONACYT (CICCO, 2021), Google (Google, 2021a), Google Académico (Google, 2021b), Web of Science (Clarivate, 2021), portales del Banco Mundial (Banco Mundial, 2021), Banco Central del Paraguay (BCP, 2021), Ministerio de Agricultura y Ganadería (MAG, 2021), Servicio Nacional de Sanidad Vegetal (SENAVE, 2021a), Instituto Nacional de Biotecnología Agrícola (INBIO, 2021a), Cámara Paraguaya de Exportadores y Comercializadores de Cereales y Oleaginosas (CAPECO, 2021b), periódicos nacionales, páginas web de empresas, páginas web de entes estatales, páginas web de entes internacionales relacionados a la producción agrícola, entre otras. Se realizaron además consultas vía correo electrónico a la Dirección de Vigilancia del SENAVE, comunicación telefónica.

En una primera búsqueda se combinaron las palabras clave y conectores para la búsqueda en todos los campos: "patógenos fúngicos", "enfermedades fúngicas soja Paraguay", "Glycine max", "producción soja Paraguay", "rendimiento soja Paraguay", donde se obtuvieron resultados desde el año 1993 al
2021.Posteriormente se utilizaron los criterios de inclusión como el año (Periodo 2016-2021) para la producción y rendimientos, idioma (inglés, español y portugués), además nombres científicos de hongos fitopatógenos reportados en soja en Paraguay, "Cercospora", "Macrophomina" "Phakop sora" nombres de enfermedades fúngicas en soja en Paraguay "Roya soja", "MOR", "Pudrición carbonosa" "Muerte súbita" entre otras enfermedades de este cultivo que se disponibilizan en la Tabla 1, además los materiales utilizados fueron los encontrados en cada plataforma de libre acceso utilizando los filtros de cada plataforma (con esa opción) y los criterios de exclusión como que presenten el término "bacterias", "virus", "insectos". Luego se seleccionaron todos los materiales técnicos y científicos disponibles en estas bases de datos que refieren a enfermedades fúngicas en soja en Paraguay, redactados en los idiomas español, portugués e inglés, durante el periodo de tiempo del 1993 al 2021.

\section{RESULTADOS}

En la Tabla 1 se presentan los hongos fitopatógenos, porcentajes de pérdidas atribuidos, etapa de aparición en el cultivo y condición climática asociada al cultivo de soja en Paraguay. Se indica además la fuente de la cual se obtuvieron los datos y si se trata de un reporte científico o técnico. 
Tabla 1. Hongos fitopatógenos en el cultivo de soja (Glycine max) en Paraguay.

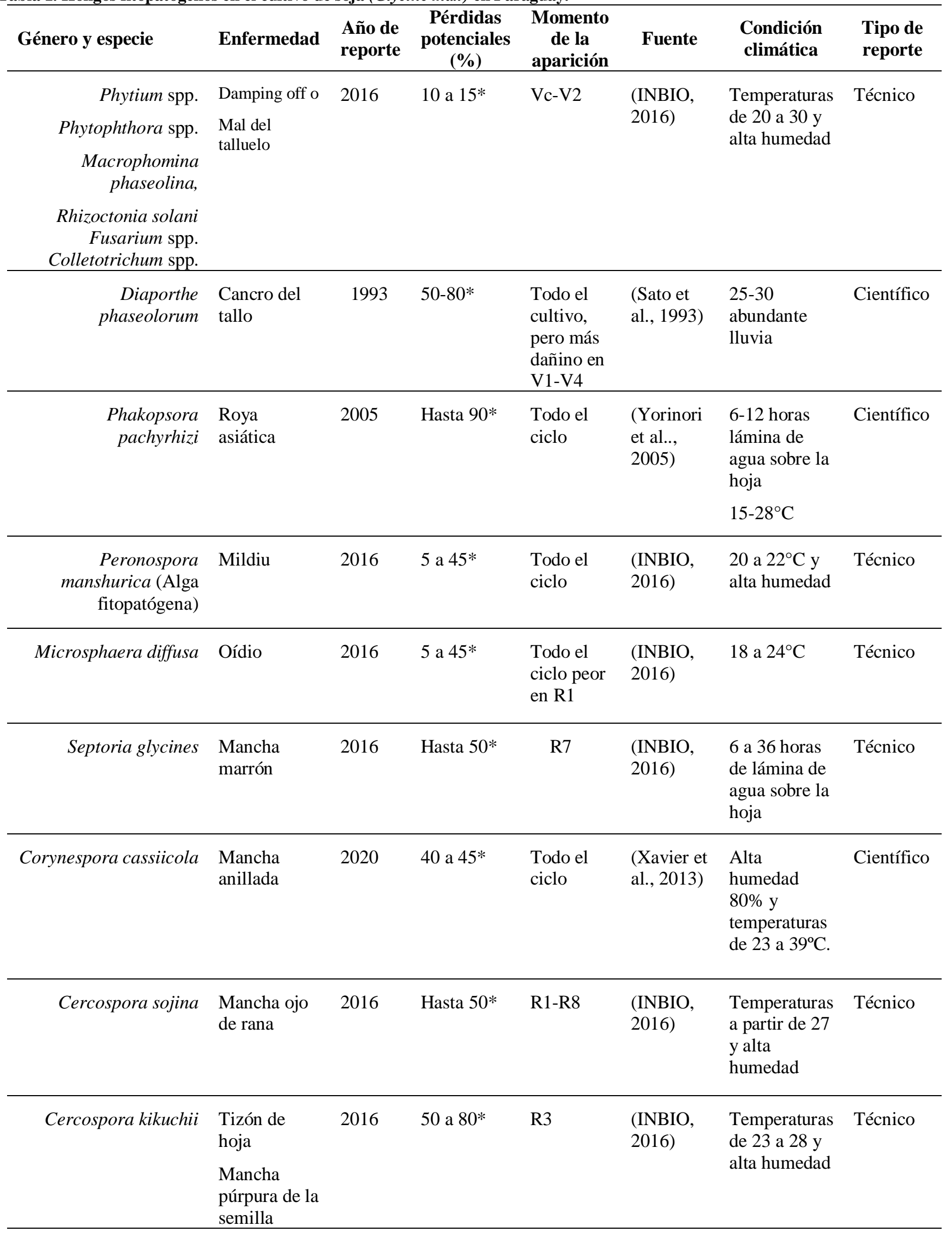


Continúa Tabla 1

\begin{tabular}{|c|c|c|c|c|c|c|c|}
\hline Género y especie & Enfermedad & $\begin{array}{l}\text { Año de } \\
\text { reporte }\end{array}$ & $\begin{array}{l}\text { Pérdidas } \\
\text { potenciales } \\
(\%)\end{array}$ & $\begin{array}{l}\text { Momento } \\
\text { de la } \\
\text { aparición } \\
\end{array}$ & Fuente & $\begin{array}{l}\text { Condición } \\
\text { climática }\end{array}$ & $\begin{array}{l}\text { Tipo de } \\
\text { reporte }\end{array}$ \\
\hline $\begin{array}{r}\text { Colletotrichum } \\
\text { truncatum }\end{array}$ & Antracnosis & 2016 & Hasta $50 *$ & R7 -R8 & $\begin{array}{l}\text { (INBIO, } \\
2016)\end{array}$ & $\begin{array}{l}\text { Temperatura } \\
\text { elevada y } \\
\text { periodos de } \\
\text { alta humedad }\end{array}$ & Técnico \\
\hline $\begin{array}{r}\text { Macrophomina } \\
\text { phaseolina }\end{array}$ & $\begin{array}{l}\text { Pudrición } \\
\text { carbonosa de } \\
\text { la raíz }\end{array}$ & 2008 & Hasta $100^{*}$ & R5-R7 & $\begin{array}{l}\text { (Ramirez } \\
\text { \& Orrego, } \\
\text { 2008) }\end{array}$ & $\begin{array}{l}\text { Temperaturas } \\
\text { superiores a } \\
30 \text { y sequía }\end{array}$ & Científico \\
\hline $\begin{array}{r}\text { Fusarium spp. } \\
\text { Fusarium solani } \\
\text { Fusarium } \\
\text { vergueliforme }\end{array}$ & $\begin{array}{l}\text { Síndrome de } \\
\text { muerte } \\
\text { súbita }\end{array}$ & 2016 & 50 a $80^{*}$ & R1 a R8 & $\begin{array}{l}\text { (Scholtz, } \\
\text { 2020) }\end{array}$ & $\begin{array}{l}\text { Temperaturas } \\
\text { de } 25 \text { a } 30 \text { y } \\
\text { alta humedad }\end{array}$ & Técnico \\
\hline
\end{tabular}

Fusarium tucumaniae

Fusarium brasiliensis

Fusarium

crassipitantum

*(INBIO, 2016; Scholtz, 2020).

Además, en la base de datos de SENAVE, se mencionan los siguientes hongos:

Ascochyta sojae, Passalora sojina, Fusarium oxysporum, Rosellinia bunodes, Rosellinia necatrix, Diaphorte phaseolorum f.sp. meridionalis, Diaphorte phaseolorum var. Sojae, Rhizoctonia solani, Phytophthora megasperma, Sclerotium rolfsii, Sclerotinia sclerotiorum, Phomopsis sojae, Phomopsis phaseoli f. sp. meridionalis (SENAVE, 2021b). El listado de hongos fitopatógenos se reproduce de manera textual.

El Banco Mundial, en su informe titulado “Análisis de riesgo del sector agropecuario en
Paraguay: Identificación, priorización, estrategia y plan de acción", menciona que para la cadena productiva de la soja, el principal riesgo asociado a la producción es la sequía de los meses de verano, principalmente en enero. El mismo documento, también menciona que si se consideran las cadenas productivas de soja, trigo y maíz, el principal riesgo asociado a la producción está dado por malezas y otras plagas y enfermedades (Banco Mundial, 2014).

Se ha indicado que actualmente las enfermedades fúngicas no representan un problema en este cultivo excepto en ciclos de cultivo o temporadas agrícolas cuando las condiciones climáticas son propicias

Tabla 2. Reducción estimada de los rendimientos de soja (miles de toneladas métricas) por enfermedades fúngicas adaptado de A. Wrather et al., 2010 y Wrather et al., 2001.

\begin{tabular}{|c|c|c|}
\hline Enfermedad* & Reporte 2001** & Reporte 2010**** \\
\hline $\begin{array}{r}\text { Antracnosis } \\
\text { Colletotrichum truncatum }\end{array}$ & 0,5 & 0,3 \\
\hline
\end{tabular}

Continúa Tabla 2 
Continúa Tabla 2

\begin{tabular}{|c|c|c|}
\hline Enfermedad* & Reporte 2001** & Reporte 2010*** \\
\hline $\begin{array}{l}\text { Mancha marrón } \\
\text { Septoria glycines }\end{array}$ & 120,0 & 0,1 \\
\hline $\begin{array}{r}\text { Pudrición carbonosa } \\
\text { Macrophomina phaseolina }\end{array}$ & 180,0 & 1,6 \\
\hline Decaimiento de la semilla por Phomopsis+ & 0,5 & Sin datos \\
\hline $\begin{array}{r}\text { Tizón de la vaina y el tallo } \\
\text { Diaporthe phaseolorum var. sojae }\end{array}$ & 0,6 & 0,1 \\
\hline $\begin{array}{r}\text { Mildew polvoriento } \\
\text { Microsphaera diffusa }\end{array}$ & 80,0 & 0 \\
\hline $\begin{array}{r}\text { Mancha púrpura de la semilla } \\
\text { Cercospora kikuchii } \\
\end{array}$ & 115,0 & 0,7 \\
\hline $\begin{array}{r}\text { Pudrición del tallo por Sclerotinia } \\
\text { Sclerotinia sclerotiorum } \\
\end{array}$ & 1,0 & 0 \\
\hline $\begin{array}{r}\text { Enfermedad de la semilla } \\
\text { Alternaria spp., Cercospora spp., } \\
\text { Corynespora sp. Cladosporium sp., } \\
\text { Phomopsis sp. Fusarium spp. }\end{array}$ & 0,5 & 0,1 \\
\hline $\begin{array}{r}\text { Cancro del tallo } \\
\text { Diaporthe phaseolorum var. caulivora and } \\
\text { var. meridionalis }\end{array}$ & 1,5 & 0 \\
\hline $\begin{array}{r}\text { Roya de la Soja } \\
\text { Phakopsora pachyrhizi }\end{array}$ & Sin datos & 1,9 \\
\hline
\end{tabular}

para el desarrollo de los hongos. Entre los patógenos que ocasionalmente podrían ocasionar problemas se menciona a los hongos Macrophomina sp., pero asociados a Fusarium sp. y nemátodos. (INBIO, 2016; Scholtz, 2020).

Otros patógenos, citados en el listado del SENAVE (SENAVE, 2021b), como por ejemplo Sclerotinia, no poseen reporte científico de su presencia en el país.

Por otra parte, Wrather y colaboradores en dos revisiones, realizadas en los años 1998 y publicado en 2001 y 2006, publicado en 2010 mencionan estiman las pérdidas por enfermedades fúngicas en diversos porcentajes que se indican en la Tabla 2.
(Wrather et al., 2010; Wrather et al., 2001).

En el reporte del año 2001, con datos del año 1998, se indica que las principales enfermedades que afectaban a la soja en Paraguay eran la mancha marrón, tizón foliar por Cercospora, el mildew polvoriento mildiu, y especialmente la pudrición carbonosa. En menor intensidad, enfermedades tardías como la mancha marrón y el tizón de la hoja por Cercospora podían reducir los rendimientos hasta en un $20 \%$ (Wrather et al., 2001).

Los mismos autores indican que entre 1997-1998, la pudrición carbonosa causó pérdidas de rendimiento de hasta un $50 \%$ en muchos campos.

Otras enfermedades que ocasionalmente 
provocaron reducción del rendimiento en Paraguay son mencionadas en este trabajo fueron la antracnosis, la pudrición de semillas por Phomopsis, la pudrición de raíz y tallo por Rhizoctonia sp. y el tizótan del sur (Wrather et al., 2001).

En cuanto a los reportes del año 2006, indican que enfermedades foliares tardías causadas principalmente por Cercospora kikuchii y Septoria sp. redujeron los rendimientos hasta en un $15 \%$ en algunos campos en 2006. El cancro del tallo, causado por Diaporthe phaseolorum var. meridionalis, se convirtió en una de las enfermedades más graves de la soja tras el primer brote en el otoño de 1992, cuando redujo el rendimiento hasta en un $50 \%$ en algunos campos (Wrather et al., 2010).

La roya de la soja se confirmó por primera vez en Paraguay durante el otoño de 2001, y las pérdidas de rendimiento alcanzaron el $60 \%$ en algunos campos. Algunos productores utilizaron cultivares resistentes, pero la resistencia no fue duradera debido a la variabilidad en el patógeno.

Las pérdidas de rendimiento fueron mayores en las variedades sembradas tardíamente. La pudrición carbonosa ha redujo el rendimiento, pero la incidencia y la severidad fueron erráticas $\mathrm{y}$ favorecidas por la sequía e indican que fue una enfermedad importante en 1996, 2001, 2005 y 2006 cuando el cultivo se vio afectado por la sequía (Wrather et al., 2010).

Los autores de estos trabajos mencionan que los métodos utilizados para estimar las pérdidas por enfermedades de la soja se realizaron estudios de campo sistemáticos, ensayos de cultivares, y cuestionarios enviados a los trabajadores de campo y al personal de extensión. Las pérdidas se basaron en estimaciones de rendimiento en ausencia de enfermedad, sin embargo estos datos no se encuentran disponibles para su verificación (Wrather et al., 2010; Wrather et al., 2001).

Posterior a esos trabajos, no se han encontrado estudios similares con datos de Paraguay. Determinar la importancia relativa de las diversas enfermedades de la soja regionalmente es fumamental. Con el tiempo con estos datos tener criterios para para equipar y apoyar a los investigadores, fitomejoradores y especialistas en extensión (Allen et al., 2017).

Sin embargo, se han realizado y publicado otro tipo de ensayos en los últimos años, entre los que se pueden mencionar "Control químico de la roya de la soja (Phakopsora pachyrhizi) con diferentes fungicidas y estados fenológicos de la planta" (Ramírez \& Orrego, 2008) "Effect of Fungicide and Timing of Application on Soybean Rust Severity and Yield" (Mueller et al., 2009), "Incidencia, severidad y control químico de la roya asiática (Phakopsora pachyrhizi Sydow) en cultivares convencionales de soja" (Aguayo Trinidad et al., 2010) "Fungicidas sitio-específicos combinados con Mancozeb para el control de la roya asiática de la soya" (EncisoMaldonado et al., 2019) y “Cuantificación de inóculo de Macrophomina phaseolina (Tassi) Goid en parcelas de soja del Departamento de ItapúaParaguay (Maidana Ojeda et al., 2020). Ninguno de estos estudios se refiere específicamente a la evaluación de daño de enfermedades fúngicas y a pesar de ser interesantes aportes no pueden utilizarse como base para estimar pérdidas.

Las variedades nacionales denominadas SOJAPAR incluyen SOJAPAR R34, precoz, SOJAPAR R19, SOJAPAR R24, SOJAPAR R49, semi precoces, y SOJAPAR R75, de ciclo medio.

Estas variedades poseen un gen (Rpp4) de resistencia a la roya asiática, por lo cual se 
recomienda hacer una aplicación de fungicida en forma preventiva; también son moderadamente resistentes a pudrición carbonosa de la raíz; resistentes a cancro de tallo y moderadamente resistentes a mancha púrpura (INBIO, 2021b; INBIO, 2021c; INBIO, 2021d; INBIO, 2021e; INBIO, 2021f).

No se encontraron disponibles los datos acerca de superficie sembrada por distintas variedades o rendimientos por variedad, tampoco encuestas a productores, pérdidas por enfermedades fúngicas en las diferentes variedades y regiones entre otros necesarios para las estimaciones de pérdidas. Con los datos obtenidos en la literatura no fue posible el cálculo de la estimación de pérdidas relacionadas a las enfermedades fúngicas en soja.

\section{DISCUSIÓN}

Dada la importancia del cultivo de soja y que el país es altamente dependiente se torna fundamental tomar las acciones necesarias para su protección y uno de los puntos fundamentales es el adecuado manejo de las enfermedades fúngicas (Ojeda, 2021).

Wrather y colaboradores, en una revisión realizada en el año 1998, mencionan que fue sólo después del brote de cancro del tallo en 1993 que las enfermedades obtuvieron cierto reconocimiento. Indican además que el desarrollo de la enfermedad de la soja en Paraguay es favorecido por el monocultivo de soja, altas poblaciones de plantas y la adopción generalizada de la labranza cero sin la rotación de cultivos, y asumen que la mayoría de las enfermedades que ocurren en Brasil también dañan el cultivo en Paraguay (Wrather et al., 2001).

La reducción de las pérdidas económicas en la producción agrícola debido a factores bióticos es de suma importancia en los sistemas agrícolas intensivos que se utilizan en la actualidad. Mantener los niveles de producción exige idear nuevas estrategias para mitigar los efectos nocivos de estos factores adversos, ya que tienen el potencial de reducir sustancialmente los rendimientos (Gharde, Singh, Dubey, \& Gupta, 2018). Como se ha mencionado en este escrito, la soja es el producto de mayor importancia para el Paraguay; en 2020, se alcanzó un récord de producción con 10.680.000 Tn, y un rendimiento medio de $2960 \mathrm{~kg} / \mathrm{Ha}$ (Abc Color, 2020). En 2020, exportación de soja representó un ingreso de divisas de 2.146 millones de dólares estadounidenses, $36 \%$ más que lo ingresado en el 2019 (PLUS, 2021), por lo cual estimar las pérdidas que pueden afectar a este cultivo se torna prioritario para el país.

Un aspecto importante es la previsibilidad del riesgo, referido a la intensidad y frecuencia de las amenazas que determinan su importancia para el sector (FAO \& IICA, 2017). Los principales factores que afectan a la producción incluyen variaciones climáticas extremas, brotes de enfermedades $\mathrm{y}$ ataque de plagas además de los costos de los insumos importados, como fertilizantes y pesticidas (Banco Mundial, 2014).

Las estimaciones de pérdidas por enfermedad se recopilan a través de varios medios que incluyen encuestas sobre enfermedades; interacciones con extensión, universidad, gobierno, industria y agricultores y experiencia personal de los productores, técnicos e investigadores con enfermedades en plantas y las pérdidas ocasionadas por las mismas (Allen et al., 2017). En Paraguay, la falta de estos datos o su acceso de manera sencilla a los investigadores, técnicos y productores 
imposibilita la estimación del riesgo de pérdidas.

Un punto fundamental detectado en este estudio es la falta de un listado actualizado de patógenos fúngicos de soja. Las fuentes oficiales como SENAVE o el Ministerio de Agricultura y Ganadería (MAG) no cuentan con la información necesaria para la realización de los cálculos. Las enfermedades como Roya Asiática de la Soja son consideradas como Emergencias o alertas fitosanitarias, sin embargo los resultados de estos monitoreos no están disponibles para el público (SENAVE, 2021a).

Por otra parte, en el listado proveído por la dirección de Vigilancia de SENAVE no se detalla cómo fueron obtenidos los reportes de esos patógenos (SENAVE, 2021b).

La importancia de las enfermedades varía anualmente, y muchos factores influyen en qué enfermedades afectan significativamente el rendimiento de los cultivos en un determinado año agrícola. Estos factores incluyen al ambiente y las condiciones climáticas, las prácticas de producción y la susceptibilidad de las variedades a las enfermedades (Allen et al., 2017).

La implementación en nuestro país de iniciativas similares al Crop Protection Network (CPN), una red multi estatal e internacional de colaboración de extensión universitaria y provincial, con especialistas y profesionales públicos y privados que proporcionan información imparcial basada en la investigación, a los agricultores y personal agrícola. Su objetivo es comunicar y ayudar a los profesionales a identificar y gestionar las enfermedades de los cultivos (Crop Protection Network, 2021) o la US Wheat and Barley Scab Iniciative (SCABUSA), que se enfoca en mejorar la seguridad alimentaria mediante la reducción del impacto de la Fusariosis de la Espiga en el trigo y la cebada y que cuenta con información para investigadores, mejoradores, productores e industria (SCABUSA, 2021). La red CPN cuenta inclusive con dos aplicaciones, una que permite el cálculo de la severidad de las enfermedades en soja y otra que permite la estimación de las pérdidas (Crop Protection Network, 2021).

Considerando la importancia de la soja en el país, es fundamental el apoyo a todas las iniciativas que permitan generar información que sea utilizada en el manejo de este cultivo, no sólo para los productores dedicados a este rubro, sino para la economía general del Paraguay. Se debe tener en cuenta que la estimación de las pérdidas de los cultivos es fundamental, ya que cuantificar los daños y compararlos con los umbrales económicos permite una adecuada toma de decisiones, determinando si un tratamiento es o no necesario y cuándo; qué se hará el próximo año, qué tipos de rotaciones de cultivos, la selección de las variedades, el tipo de manejo que se hará de las enfermedades, entre otros (Breunig, 2021).

Por otra parte, es importante mencionar que para la formulación de políticas adecuadas es necesario que las estimaciones de pérdidas no sean generales y poco sistemáticas, puesto que para orientar las decisiones a nivel nacional o local de debe contar con datos fidedignos (Teng et al., 1989).

Teng y colaboradores mencionan que para ayudar a los científicos a anticipar cambios en la productividad en el futuro, y para desarrollar estrategias de manejo de enfermedades que contribuyan a un desarrollo sostenido y estable rendimientos es fundamental el conocimiento del comportamiento de las enfermedades a lo largo del tiempo en la misma zona (Teng et al., 1989). Otro punto fundamental es que mantener la salud de las 
plantas, entender el efecto y comportamiento de los patógenos en campo y sus efectos cuantitativos en los cultivos es esencial para evaluar la eficacia de las prácticas de manejo del cultivo, lograr la estabilidad de los sistemas de producción, evaluar la efectividad de los sistemas y servicios regulatorios y mantener la seguridad alimentaria de las familias (Cerda et al., 2017).

Un aspecto positivo identificado en este trabajo es que las variedades nacionales, según sus descripciones, presentan ventajas comparativas con respecto a las enfermedades a las que son resistentes y moderadamente resistentes. Su adaptación a las condiciones agroclimáticas del país (INBIO, 2021b, 2021c, 2021d, 2021e, 2021f) y el uso podría llevar a una reducción del gasto en fungicidas (IshikawaIshiwata \& Furuya, 2021). Sumado a esto es necesario otorgar a los productores y técnicos de campo, las herramientas que permitan el desarrollo del cultivo y una producción exitosa.

\section{CONCLUSIÓN}

Se puede concluir que a pesar de la importancia de la soja en la economía nacional, con los datos disponibles públicamente, no es posible la estimación de pérdidas de rendimiento de soja ( $G$. max) por enfermedades fúngicas a nivel país. Es de suma necesidad generar datos fidedignos que puedan ser utilizados para la previsión de las acciones a tomar en referencia a las enfermedades presentes en los sojales. También se visualiza la necesidad de un trabajo coordinado entre productores, industria, gobierno y academia.

\section{AGRADECIMIENTO}

Se agradece la colaboración a la Ing. Agr. MSc. Ruth Scholtz, Ing. Agr. Pablo Arrúa, Ing. Agr. Darío Flecha, Ing. Agr. Oscar Benítez.

Declaración de interés. Los autores declaran no tener conflicto de intereses.

\section{REFERENCIAS BIBLIOGRÁFICAS}

Abc Color. (2019). Riesgos agrícolas en Paraguay, en la visión del Banco Mundial. $A B C$ Color Suplemento Económico.https://www.abc.com.py/edicionimpresa/suplementos/economico/riesgos-agricolas-enparaguay-en-la-vision-del-banco-mundial-1732498.html

Abc Color. (2020). Cosecha récord de soja opacada por bajos precios. Abc Color. Nacionales.

https://www.abc.com.py/nacionales/2020/07/06/cosecharecord-de-soja-opacada-por-bajos-precios/

Aguayo Trinidad, S., Orrego Fuente, A. L. \& Rodríguez Espínola, H. N. (2020). Incidencia, severidad y control químico de la roya asiática (Phakopsora pachyrhizi Sydow) en cultivares convencionales de soja. Investigación Agraria, 12(1), 11-16. http://www.agr.una.py/revista/index.php/ria/article/view/ 13

Allen, T. W., Bradley, C. A., Sisson, A. J., Byamukama, E., Chilvers, M. I., Coker, C. M., Coker, C. M., Collins, A. A., Damicone, J. P., Dorrance, A. E., Dufault, N. S., Esker, P. D., Faske, T. R., Giesler, L. J., Grybauskas, A. P., Hershman, D. E., Hollier, C. A., Isakeir, T., Jardine, D. J....Allen Wrather, J. (2017). Soybean yield loss estimates due to diseases in the United States and Ontario, Canada, from 2010 to 2014. Plant Health Progress, 18(1), 19-27. https://doi.org/10.1094/PHP-RS-16-0066

Banco Mundial. (2014). Análisis de riesgo del sector en Paraguay. https://documentos.bancomundial.org/es/publ ication/documents-

reports/documentdetail/105821468332711721/análisisde-riesgo-del-sector-agropecuario-en-paraguayidentificación-y-priorización-de-los-riesgosagropecuarios

Banco Mundial. (2018). Paraguay - Notas de Política Paraguay. Notas de Política 2018. Noticias Del Banco Mundial. http://documentos.bancomundial.org/curated/es/7510715 25763871071/pdf/126021-WP-PUBLIC-SPANISHPYNotasdePolticafinal.pdf

Banco Mundial. (2021). Banco Mundial. https://www.bancomundial.org/es/home

BCP. (2021). Banco Central del Paraguay. https://www.bcp.gov.py/

Breunig, M. (2021). General Scouting Tips and Safety Crop Protection Network Virtual Regional Crop Scout School. Michigan. Crop Protection Network. https://cropprotectionnetwork.s3.amazonaws.com/webinars/general-scoutingtips-and-safety-presentation_file-2021-04-15-172842.pdf 
CAPECO. (2019a). Quiebre de la producción de soja asciende a $23,5 \%$ y pérdidas económicas a USD 1340 millones. https://capeco.org.py/2019/05/16/quiebre-de-laproduccion-de-soja-asciende-a-235-y-perdidaseconomicas-a-usd-1340-millones/

CAPECO. (2019b). Situación de la cosecha, producción de soja de la campaña 2018-2019 y sus implicancias. http://capeco.org.py/wpcontent/uploads/2019/05/Estimacion-de-producciónsoja-campaña-2018-2019-Capeco-UGP.pdf

CAPECO. (2021a). Área de siembra, producción rendimiento. https://capeco.org.py/area-de-siembraproduccion-y-rendimiento/

CAPECO. (2021b). Cámara Paraguaya de Exportadores y Comercializadores de Cereales y Oleaginosas. https://capeco.org.py/

Cerda, R., Avelino, J., Gary, C., Tixier, P., Lechevallier, E. \& Allinne, C. (2017). Primary and secondary yield losses caused by pests and diseases: Assessment and modeling in coffee. PLOS ONE,12. https://doi.org/10.1371/journal.pone.0169133

CICCO. (2021). Portal de Acceso a Información Científica del Consejo Nacional de Ciencia y Tecnología del Paraguay. https://cicco.conacyt.gov.py/

Clarivate. (2021). Web of Science Master Journal List. https://mjl.clarivate.com/search-results

Crop Protection Network (2021). Crop Protection Network. https://cropprotectionnetwork.org/

Enciso-Maldonado, G. A., Maidana-Ojeda, M., SchlickmannTank, J. A., Montoya-García, C. O., Páez-Ranoni, H. J., Fernández-Riquelme, F. \& Domínguez-Sanabria, J. A. (2019). Fungicidas sitio-específicos combinados con Mancozeb para el control de la roya asiática de la soya. Revista Mexicana de Fitopatología, Mexican Journal of Phytopathology, 37(1). https://doi.org/10.18781/r.mex.fit.1903-3

FAO \& IICA. (2017). Gestión integral del riesgo de desastres en el sector agrícola y la seguridad alimentaria en los países del CAS. FAO. http://www.fao.org/3/a-i8158s.pdf

Gharde, Y., Singh, P. K., Dubey, R. P. \& Gupta, P. K. (2018). Assessment of yield and economic losses in agriculture due to weeds in India. Crop Protection, 107, 12-18. https://doi.org/10.1016/j.cropro.2018.01.007

Google. (2021a). Google. https://www.google.com/webhp?hl=es419\&sa=X\&ved=0ahUKEwjU04Os9YfxAhVJJrkGHUo pCQcQPAgI

Google. (2021b). Google Académico. https://scholar.google.es/schhp?hl=es

INBIO. (2016). Guia de campo: Identificacion malezas, plagas y enfermedade de soja. Asunción. INBIO. https://www.inbio.org.py/publicaciones/

INBIO. (2021a). Instituto de Biotecnología Agrícola. https://www.inbio.org.py/

INBIO. (2021b). SOJAPAR RI9. https://www.inbio.org.py/sojapar/sojapar-r19/

INBIO. (2021c). SOJAPAR R24. https://www.inbio.org.py/sojapar/sojapar-r24/

INBIO. (2021d). SOJAPAR R34. https://www.inbio.org.py/sojapar/sojapar-r34/

INBIO. (2021e). SOJAPAR R49. https://www.inbio.org.py/sojapar/sojapar-r49/

INBIO. (2021f). SOJAPAR R75. https://www.inbio.org.py/sojapar/sojapar-r75/

Ishikawa-Ishiwata, Y. \& Furuya, J. (2021). Fungicide cost reduction with soybean rust-resistant cultivars in Paraguay: A supply and demand approach. Sustainability
(Switzerland), 13, 1-14. https://doi.org/10.3390/su13020887

MAG. (2021). Ministerio de Agricultura y Ganadería. http://www.mag.gov.py/

Maidana Ojeda, M., Sosa, J., Bogado González, C., Fernández, M., Sanabria Velázquez, A. D., López Nicora, H. D., \& Enciso Maldonado, G. A. (2020). Cuantificación de inóculo de Macrophomina phaseolina (Tassi) Goid en parcelas de soja del Departamento de Itapúa-Paraguay. Investigaciones y Estudios - UNA, 11(2), 69-77. https://doi.org/10.47133/ieuna2027

Mueller, T. A., Miles, M. R., Morel, W., Marois, J. J., Wright, D. L., Kemerait, R. C., Levy, C. \& Hartman, G. L. (2009). Effect of Fungicide and Timing of Application on Soybean Rust Severity and Yield. Plant Disease, 93(3), 243-248. https://doi.org/doi:10.1094/ PDIS-93-30243

Ojeda, M. (2021). Manejo integrado de enfermedades de soja en Paraguay. Actualidad y perspectivas. Rainbow Paraguay, 1 . https://www.rainbowagroconosur.com/py/detalle-demanejo-integrado-de-enfermedades-de-soja-enparaguay-actualidad-y-perspectivas227?fbclid=IwAR1wgg3nfSNiWuQIhmFa4ZWG49mbz 1YuPjcHAGFaYiQPZUjpL4acU3-Opzw

Ramirez, Y. \& Orrego, A. (2008). Eficiencia de diferentes métodos de inoculación artificial de Macrophomina phaseolina en soja (Glycine max.). Investigación Agraria, 10(2), 66-71. http://www.agr.una.py/revista/index.php/ria/article/view/ 54.

PLUS. (2021). Exportaciones del complejo soja generan US\$ 3.971 millones en 2020. Finanzas, 1 . https://www.revistaplus.com.py/2021/01/19/exportacion es-del-complejo-soja-generan-us-3-971-millones-en2020/

Sato, T., Viedma, L. Q. De, Alvarez, E., Romero, M. I. \& P, W M. (1993). First occurrence of soybean southern stem canker in Paraguay. Japan Agricultural Research Quarterly, 27(February 1992), 20-20. https://www.jircas.go.jp/sites/default/files/publication/jar q/27-1-020-026 0.pdf

SCABUSA. (2021). US Wheat and Barley Iniciative. https://scabusa.org/

Scholtz, R. (2020). Identificación de las principales enfermedades en el cultivo de Soja, Manual Técnico: edición especial IPTA 10 años. Capitán Miranda. IPTA. https://ipta.gov.py/application/files/6615/9733/8663/Enf ermedades_Soja_Rut_IPTA2020_compressed.pdf

SENAVE. (2021a). Servicio Nacional de Sanidad Vegetal y de Semillas. https://www.senave.gov.py/mapa-proteccionvegetal

SENAVE (2021b). Plagas presentes en el cultivo de soja en Paraguay. Asunción.

Teng, P. S., Mew, T. W., Bonman, J. M. \& Leung, H. (1989). Methods for estimating yield loss in tropical rice diseases. Tropical Agriculture Research Series, (22), $141-152$

Wrather, A., Shannon, G., Balardin, R., Carregal, L., Escobar, R., Gupta, G. K., Morel, Z., Ploper, D. \& Tenuta, A. (2010). Effect of Diseases on Soybean Yield in the Top Eight Producing Countries in 2006. Plant Health Progress, 11(1), 29. https://doi.org/10.1094/php-2010$\underline{0102-01-r s}$

Wrather, J. A., Anderson, T. R., Arsyad, D. M., Tan, Y., Ploper, L. D., Porta-Puglia, A. \& Yorinori, J. T. (2001). Soybean disease loss estimates for the top ten soybean- 
producing countries in 1998. Canadian Journal of Plant Pathology, 23(2), 115-

121.https://doi.org/10.1080/07060660109506918

Xavier, S. A., Canteri, M. G., Barros, D. C. M. \& Godoy, C. V. (2013). Sensitivity of Corynespora cassiicola from soybean to carbendazim and prothioconazole. Tropical Plant Pathology, 38(5), 431-435.

https://doi.org/10.1590/S1982-56762013005000020
Yorinori, J. T., Paiva, W. M., Frederick, R. D., Costamilan, L. M., Bertagnolli, P. F., Hartman, G. E., Godoy, C. V. \& Nunes, J. (2005). Epidemics of soybean rust (Phakopsora pachyrhizi) in Brazil and Paraguay from 2001 to 2003. Plant Disease, 89(6), 675-677. https://doi.org/10.1094/PD-89-0675 